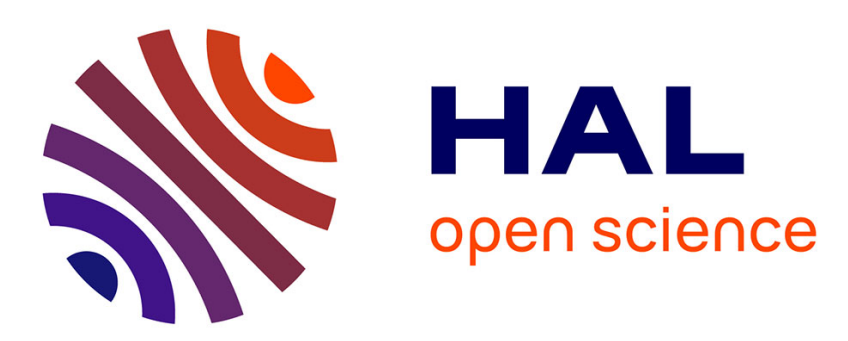

\title{
Synthesis of Goethite by Separation of the Nucleation and Growth Processes of Ferrihydrite Nanoparticles using Microfluidics
}

\author{
Ali Abou Hassan, Olivier Sandre, Sophie Neveu, Valérie Cabuil
}

\section{To cite this version:}

Ali Abou Hassan, Olivier Sandre, Sophie Neveu, Valérie Cabuil. Synthesis of Goethite by Separation of the Nucleation and Growth Processes of Ferrihydrite Nanoparticles using Microfluidics. Angewandte Chemie International Edition, 2009, 48 (13), pp.2342 - 2345. 10.1002/anie.200805933 . hal-00409238

\section{HAL Id: hal-00409238 \\ https://hal.science/hal-00409238}

Submitted on 25 May 2019

HAL is a multi-disciplinary open access archive for the deposit and dissemination of scientific research documents, whether they are published or not. The documents may come from teaching and research institutions in France or abroad, or from public or private research centers.
L'archive ouverte pluridisciplinaire HAL, est destinée au dépôt et à la diffusion de documents scientifiques de niveau recherche, publiés ou non, émanant des établissements d'enseignement et de recherche français ou étrangers, des laboratoires publics ou privés. 


\title{
Synthesis of Goethite by Separation of the Nucleation and Growth of Ferrihydrite Nanoparticles by Microfluidics**
}

\author{
Ali Abou-Hassan*, Olivier Sandre, Sophie Neveu and Valérie Cabuil
}

Continuous flow and droplets-based microreactors have been largely used for studying and optimizing chemical reactions. ${ }^{[1-13]}$ Several nanoparticles have been synthesized in microfluidic devices: Quantum Dots as $\mathrm{CdS},{ }^{[5]} \mathrm{CdSe}^{[6]} \mathrm{CdSe}-\mathrm{ZnS}$ core-shell nanoparticles, ${ }^{[7]}$ metallic nanoparticles as $\mathrm{Pd}, \mathrm{Co}, \mathrm{Ag}, \mathrm{Au},{ }^{[8]}$ and oxides as $\left.\gamma-\mathrm{Fe}_{2} \mathrm{O}_{3},{ }^{[9,}, 10\right] \mathrm{SiO}_{2},{ }^{[11]} \mathrm{TiO}_{2}{ }^{[12]}$ or $\mathrm{SiO}_{2}-\mathrm{TiO}_{2}$ core-shell nanoparticles. ${ }^{[13]}$ Compared to conventional bulk synthesis, microfluidics offers a better control of the reaction parameters (temperature, mass transfer...) which can lead to a better control of particles size. ${ }^{[1-4]}$ Goethite $(\alpha-\mathrm{FeOOH})$ is an iron oxi-hydroxide widely found in iron-rich soils. ${ }^{[14]}$ This clay mineral constitutes the natural ochre pigment and because of its elongated shape, synthetic goethite is often used as a precursor of $\alpha$-Fe "hard magnet" particles for magnetic recording. ${ }^{[15]}$. Again because of this elongated shape, suspensions of anti-ferromagnetic goethite nano-laths exhibit an original magneto-optical effect and self-assemble spontaneously into a nematic liquid crystal phase above a threshold concentration. ${ }^{[16]}$ Different methods have been reported for the synthesis of acicular goethite particles principally based on aging of the ferrihydrite nanoparticles obtained by alkalinisation of iron (III) salt solutions, ${ }^{[17]}$ but to date a microfluidic synthesis of goethite particles has not been demonstrated. Due to the importance of particles shape for the improvement of the magnetic properties or for the control of the particles assembly, controlling the synthesis conditions of these particles is important.

So-called "digital microfluidics" manipulating biphasic flows is currently very popular to produce microdroplets, behaving as microreactors for the synthesis of nanoparticles. However, it frequently uses oils and surfactants that can affect the nucleationgrowth mechanisms of the particles. Compared to microdroplets reactors, continuous flow reactors are easier to handle and more representative of the conditions of bulk synthesis with improved homogeneity so as to offer a better reproducibility of the results. ${ }^{[20]}$ Another benefit provided by the continuous microfluidic systems is the ability to add reagents all over the channel length, without

[*] Prof. V. Cabuil. Dr. O. Sandre, Dr. S. Neveu and PhD A. Abou-Hassan.

UPMC Univ Paris 6, Laboratoire des Liquides loniques et Interfaces Chargées UMR 7612, équipe Colloïdes Inorganiques (LI2C)

Université Paris 6 (UPMC) Bat $F(74)$, case 51

4 place Jussieu, F-75252 Paris Cedex 05 (France)

Fax: (+33) 1-44-27-36-75

E-mail: ali.abou_hassan@upmc.fr

Homepage : http://www.li2c.upmc.fr

[**] We thank Patricia Beaunier from the electron microscopy facility (UPMC, UFR 926) for TEM and HRTEM images, Virginie Georget from the cellular imaging facility for LCSM images (UPMC, IFR 83), Preston Mohr and Thomas Boudier (UPMC, IFR 83) for fruitful discussions about image analysis. needing to synchronize with a dropping frequency as for microdroplets reactors. ${ }^{[21]}$ Mixing in the continuous flow reactors is diffusion-limited, occurring by diffusion of the chemical species at the confluence of the flows. ${ }^{[22]}$ To overcome this limitation, hydrodynamic flow focusing based on the squeezing of a sample flow between sheath flows by using appropriate geometrical conditions has been demonstrated as a reliable method to reduce the diffusion distances and decrease the mixing times. ${ }^{[23]}$ By adjusting the volumetric rates respectively of sample and sheath flows, a stable central jet is achieved. Besides, the parabolic velocity profile due to the laminar Poiseuille flow is nearly flat in the central focalised jet flow and can be described as a plug flow, so that when the chemical reaction proceeds in the sample jet, one can expect to minimize the shear stress causing the Taylor-Aris dispersion effect during the nanoparticles synthesis. ${ }^{[24]}$

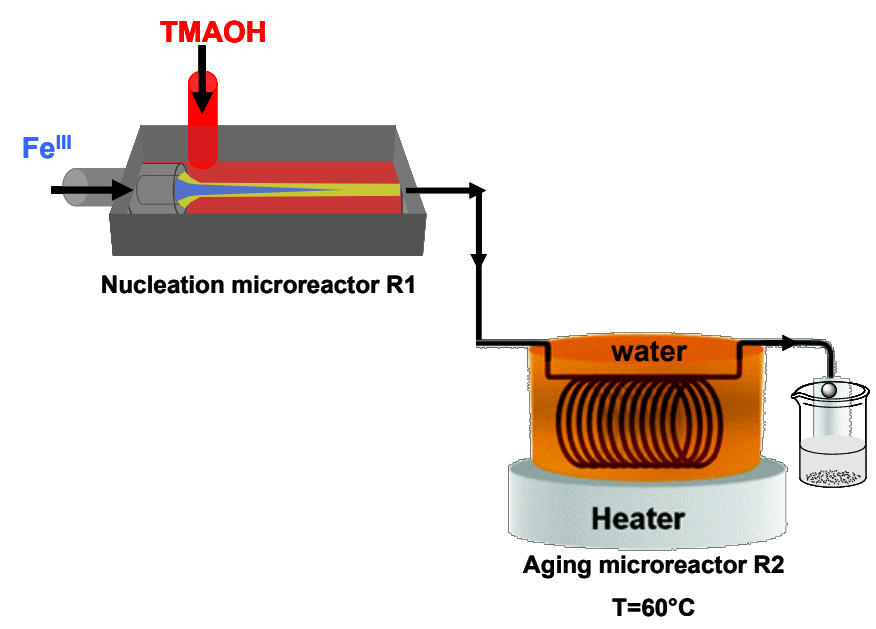

Figure 1. Schematic representation of the experimental setup used for the preparation of the ferrihydrite and goethite nanoparticles.

The aim of the present work is to describe a new method based on microfluidics allowing the acceleration and the control of the production of small goethite nanolaths. The idea is to separate the nucleation of the ferrihydrite nanoparticles from their growth into goethite particles. The nucleation of the primary nanoparticles of ferrihydrite is induced by diffusive mixing at room temperature in a microreactor based on a coaxial flows-geometry (R1; Figure 1). This mixing reactor described in a previous work ${ }^{[9]}$ is based on a three dimensional coaxial flows device of two streaming reagents. The 3D hydrodynamic geometry allows a rapid homogeneity of the reactants through focalisation and avoids technical problems such as channel clogging and fooling onto the reactor walls. 
To induce the nucleation reaction in the $\mathrm{R} 1$ microreactor, the solubilised iron (III) chloride solution and the alkaline solution of tetramethyl ammonium hydroxide (TMAOH) are flushed respectively into the inner and the outer streams with the rate flows $\mathrm{Q}_{\text {in }}$ and $\mathrm{Q}_{\text {out }}$. The rate flow of the different species can be continuously adjusted in order to produce a stable inner stream and different mixing times. Fast mixing is required to ensure a homogeneous and uniform chemical composition of the nucleation region in the microreactor.

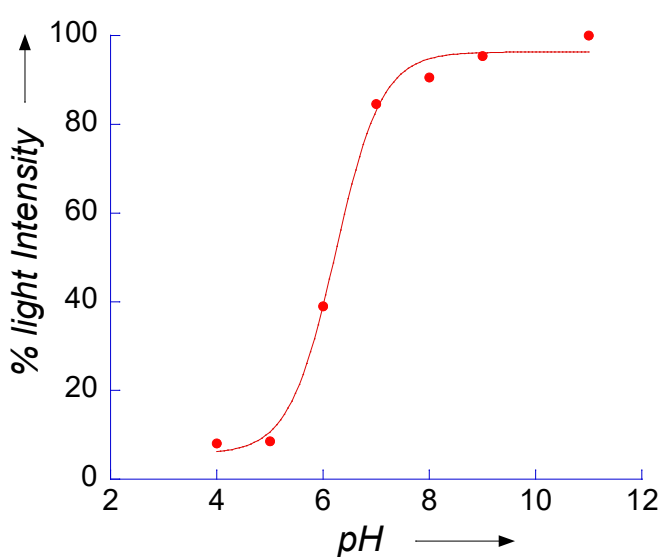

Figure 2. Fluorescence intensity of a $6 \mu \mathrm{M}$ fluorescein solution at different $\mathrm{pH}$ normalized by its maximum value.

The mixing time is determined experimentally from a 3D-mapping of $\mathrm{pH}$ inside the channel R1. A detailed physical analysis including numerical simulations will be published elsewhere. In a first approximation, mixing time by flow focusing in the laminar regime is independent of the total volumetric flow rate $\mathrm{Q}_{\text {tot }}$ and depends only on the ratio of the outer to inner flow rates $\mathrm{Q}_{\text {out }} / \mathrm{Q}_{\text {in }}$ as reported initially by Scampavia et al. ${ }^{[25]}$ However, our preliminary results indicate that increasing $\mathrm{Q}_{\mathrm{tot}}$ also has a strong effect on the polydispersity of the produced nanoparticles by decreasing the width of the residence time distribution. Experimentally, the quantitative analysis of the mixing time and the $\mathrm{pH}$ mapping in the nucleation reactor R1 is performed by labelling the acidic inner stream by a 6 $\mu \mathrm{M}$ fluorescein solution. Fluorescein is used as a $\mathrm{pH}$ probe since it can exist in several ionization forms, each one of which having distinct spectral properties. ${ }^{[26]}$ Scheme 1 and Figure 2 respectively show only the prevalent forms in aqueous solution and the experimental fluorescence intensity versus $\mathrm{pH}$ curve for $6 \mu \mathrm{M}$ solution. By following the fluorescein emission intensity inside the mixing channel R1 by confocal laser scanning microscopy (CLSM), we obtain a cross-section plot of the $\mathrm{pH}$ jump along the focalised stream jet from which we get an estimate of the mixing time by assuming a linear relationship between time and position. After some trials, the best mixing time achieved in the micromixer R1 is about $80 \mathrm{~ms}$ for a ratio Qout/Qin 400 .

Figure 3 shows the dynamical mixing in the focalised jet due to diffusion of the $\mathrm{OH}^{-}$ions flowing in the outer stream into the fluorescein central stream. The fast mixing is inferred from the steep increase of the fluorescence intensity profile along the $x$ axis corresponding to a $\mathrm{pH}$ jump from below to above the $\mathrm{pKa} \sim 6.2$ of fluorescein, before reaching a plateau value for an alkaline $\mathrm{pH}$ around 13 (due to the final concentration of TAMOH). As $\mathrm{pH}$ rises above neutrality, the solubility of the iron (III) species decreases and as soon as the supersaturation concentration is reached, nucleation of ferrihydrite occurs.
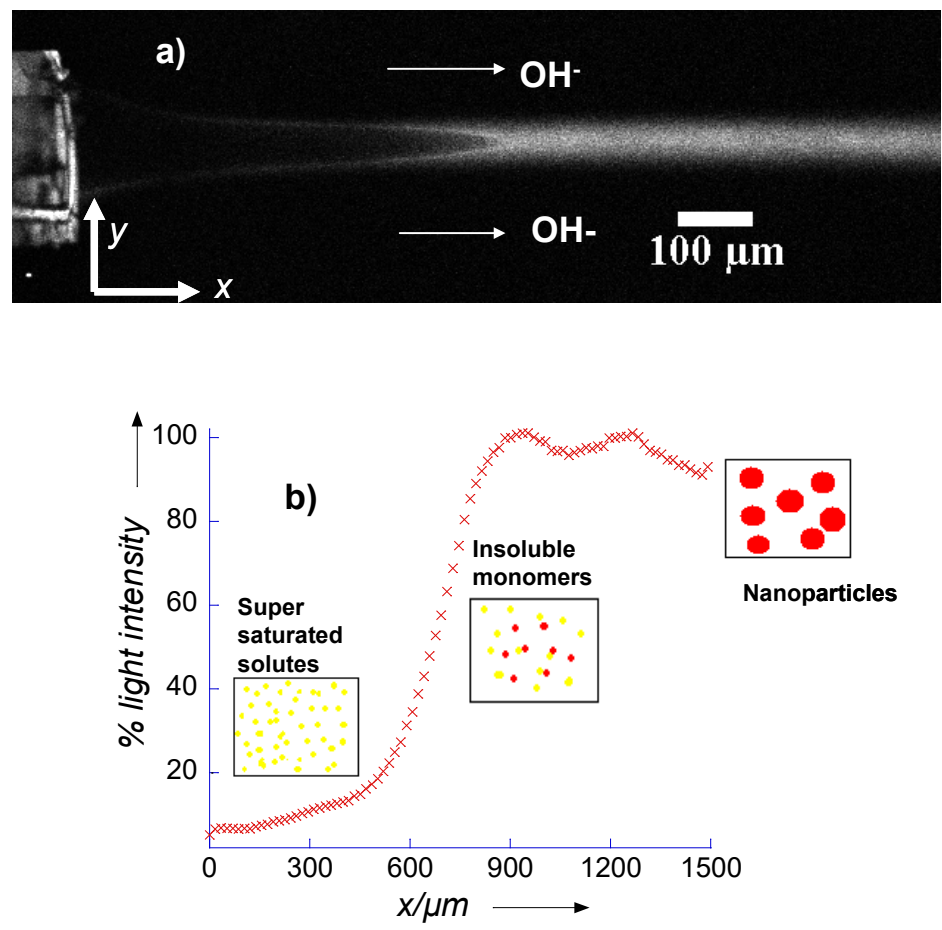

Figure 3. a) CLSM image during the mixing of the acidic inner stream labelled with $6 \mu \mathrm{M}$ fluorescein solution with the outer stream of TMAOH solution. The flow rates are $Q_{\text {in }}=0.5 \mu \mathrm{l} / \mathrm{min}$ and $Q_{\text {out }}$ $200 \mu \mathrm{l} / \mathrm{min}$. b) average plot profile of the fluorescence intensity in the inner stream as a function of position in the channel during mixing, reflecting the $\mathrm{pH}$ variation.

Before aging, a drop is collected at the outlet of R1 and analysed by Transmission Electron Microscopy (TEM). Figure 4a shows well defined spherical ferrihydrite nanoparticles (nanodots) for which particle-size measurements result into a $4 \pm 1 \mathrm{~nm}$ average particle diameter. The electron diffraction pattern of a large selected zone confirms the presence of " 2 lines" (meaning poorly crystalline) ferrihydrite. ${ }^{[17]}$ Moreover, high resolution TEM (HRTEM) measurements (Figure 4b) show that the nanoparticles are monocrystaline, exhibiting atomic planes with an interplanar distance of about $2.5 \AA$, which is consistent with ferrihydrite nanoparticles.

At the outlet of the R1 micromixer, the suspended ferrihydrite nanoparticles are directly injected into the microtubular aging coil $\mathrm{R} 2(\mathrm{~L}=150 \mathrm{~cm})$, which is continuously heated in a water bath at $60^{\circ} \mathrm{C}$. Temperature profiles are calculated in order to determine the tubing length (hence the time) needed for the fluid to reach a steady state. Under the operating flow rate, the ferrihydrite solution reaches $60^{\circ} \mathrm{C}$ in $\sim 1 \mathrm{~s}$, i.e. within the first centimeter after it entered the heated zone of the tubing. The effective resident time is about 15 min, as estimated from the tubing length along which the fluid has reached the stationary temperature of $60^{\circ} \mathrm{C}$. 


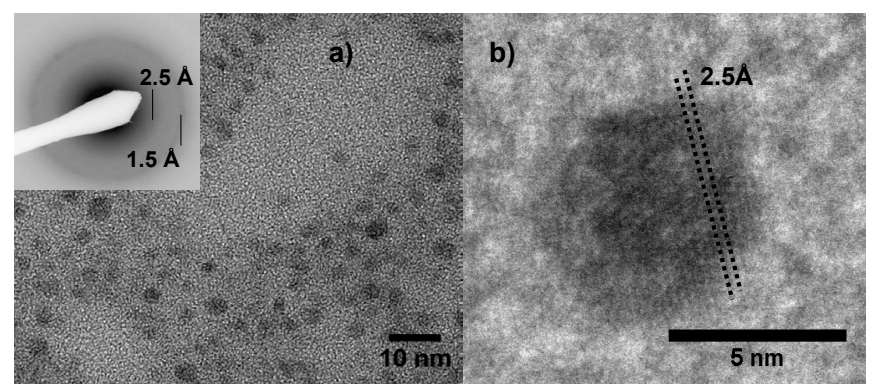

Figure 4. a) TEM picture of the sample taken after precipitation in the $\mathrm{R} 1$ microreactor (before aging) showing $4 \pm 1 \mathrm{~nm}$ diameter ferrihydrite nuclei. The selected area diffraction pattern (inset) is typical of 2-lines ferrihydrite. b) HRTEM image of an individual ferrihydrite nanoparticle with a $2.5 \AA$ lattice fringe.

After aging for 15 min under continuous flow through R2, goethite nanolaths under growth are observed with an average length $\mathrm{L}=30 \pm 17 \mathrm{~nm}$ and width $\mathrm{w}=7 \pm 4 \mathrm{~nm}$ ( $\mathrm{N}=100$ particles) yielding an axial ratio $\mathrm{L} / \mathrm{w}=4 \pm 4$ (Figure $5 \mathrm{a}$ ). This short aging time appeared to be sufficient for the growth of fairly crystalline and anisotropic goethite nanoparticles differing only by smaller sizes compared to ones obtained after complete aging (one day at $60^{\circ} \mathrm{C}$ according to literature ${ }^{[27]}$ ). Moreover, the presence of remaining ferrihydrite nuclei undergoing aggregation in the batch after $15 \mathrm{~min}$ and even after $24 \mathrm{~h}$ at $60^{\circ} \mathrm{C}$ (date not shown) supports the idea that goethite nanoparticles were apparently formed here by the aggregation mechanism rather than by dissolution/reprecipitation. We attribute (040) indexes to the lattice fringes with $2.5 \AA$ spacing running parallel all along the length of the crystal (thereby an evidence of monocrystalinity) as imaged by HRTEM (Figure 5b). In addition, the particle of Figure $5 \mathrm{~b}$ appears to be composed of nodules that are similar in size to the nanodots, which is consistent with the idea that growth proceeds by aggregation of nuclei that are aligned by the shear stress of the laminar flow.

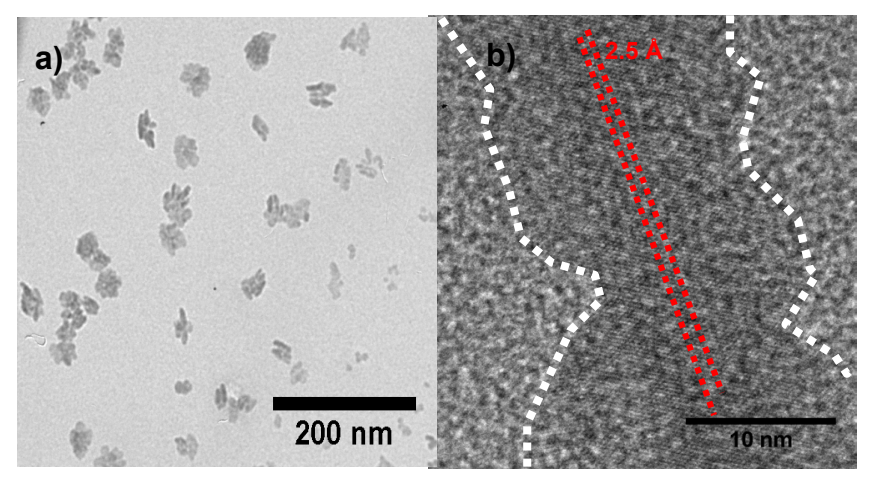

Figure 5. a) TEM image of the produced nanolaths after aging for 15 min in the R2 microtubular loop, at $\mathrm{pH}-13$ and under laminar flow b) HRTEM image of a nanorod particle. Lattice fringe spacing is consistent with goethite. The dashed lines serve to highlight the morphology and texture of the particle.

In summary, we have demonstrated a significant acceleration of the synthesis of goethite nanoparticles from ferrihydrite nuclei by the use of a continuous-flows microfluidic system. The novelty of this approach lies in the fact that nucleation of the primary particles (ferrihydrite) and growth of the goethite nanoparticles are separated in two independent microreactors operating in different conditions. In the nucleation microreactor, the streaming reagents are mixed by molecular diffusion at room temperature in a flow focusing geometry. The homogeneity of the mixture is ensured by the fast mixing time that we estimated by fluorescence microscopy. Moreover, the technical problem of microchannel clogging due to precipitation onto the walls is avoided thanks to the $3 \mathrm{D}$ geometry. Compared to bulk synthesis that yields goethite nanoparticles with typically length $\mathrm{L}=250 \mathrm{~nm}$, width $\mathrm{w}=40 \mathrm{~nm}$ and polydispersity index about $50 \%$ for both dimensions, ${ }^{[28]}$ our method of growth in a microfluidic aging channel minimizes local temperature gradients, ensures a regular laminar flow and finally leads to crystalline nanolaths with approximately the same values of aspect ratio and polydispersity index than in the bulk synthesis but smaller in sizes. Moreover, the short time required for aging (15 min for a velocity of $0.1 \mathrm{~cm} / \mathrm{s}$ ) compared to bulk synthesis (several hours or days) may originate from the small diameter of the aging reactor causing a shear that pre-aligns the primary ferrihydrite nanoparticles and speed up their oriented aggregation process. Beside the importance of the different applications of goethite, this work opens a new fundamental question about the use of microfluidics in the aging process of materials dispersed in a fluid carrier.

\section{Experimental Section}

The coaxial flows micromixer (R1) is obtained by molding in poly(dimethylsiloxane) (PDMS) and is described in details in the ref 9. The aging microtubular loop (R2) consists in a transparent PTFE tube of $1.7 \mathrm{~mm}$ inner diameter and $150 \mathrm{~cm}$ total length (Upchurch Scientific). Harvard Apparatus syringe pumps (pico 11 plus) controlled the flow rates. As starting materials for the precipitation of iron oxyhydroxide nanoparticles, we used $\mathrm{FeCl}_{3}$ (27\%, VWR), tetramethyl ammonium hydroxide $\left(\mathrm{CH}_{3}\right)_{4} \mathrm{NOH}(97 \%$, Sigma Aldrich), and hydrochloric acid $\mathrm{HCl}(37 \%, \mathrm{VWR})$ of analytical grade. For the CLSM images, fluorescein sodium salt $\mathrm{C}_{20} \mathrm{H}_{10} \mathrm{Na}_{2} \mathrm{O}_{5}(98.5 \%$, Sigma Aldrich) is used. $0.1 \mathrm{M}$ and $0.01 \mathrm{M} \mathrm{Fe}$ III solutions are prepared by dilution of the commercial solution by $0.1 \mathrm{M}$ hydrochloric acid solution. TAMOH solutions at $0.1 \mathrm{M}(\mathrm{pH} 13)$ are used rapidly after preparation to prevent carbonatation. $6 \mu \mathrm{m}$ fluorescein sodium solution is prepared by adding the fluorescein sodium salt to $0.1 \mathrm{M}$ hydrochloric acid. TEM and HRTEM images were recorded with a JEOL JEM 100CX transmission electron microscope operating at $100 \mathrm{kV}$ with a point to point resolution of $0.3 \mathrm{~nm}$. Confocal Laser Scanning Microscopy (CLSM) was performed on a Leica SP5 microscope with a 5X (O.11NA) objective. $488 \mathrm{~nm}$ argon laser line was used for excitation and emitted fluorescence collected from 500 to $600 \mathrm{~nm}$

Received: December 5, 2008

Published online: February 16, 2009

Keywords: goethite $\cdot$ microreactors $\cdot$ nucleation-growth separation . nanoparticles $\cdot$ aging

W. Ehrfeld, V. Hessel, H. Lowe, Microreactors: New Technology for Modern Chemistry, Wiley -VCH, Weinheim, 2000.

[2] K. Jähnisch, V. Hessel, H. Löwe, M. Baerns, Angew. Chem. 2004 116, 410-451; Angew. Chem. Int. Ed. 2004, 43, 406-446.

[3] K. F. Jensen, Chem. Eng. Sci. 2001, 56, 293-303.
[4] A. J. deMello, J. C. deMello, Lab Chip 2004, 4, 11N-15N.

[5] J. B. Edel, R. Fortt, J. C. deMello, A. J. deMello, Chem. Commun. 2002, 1136-1137.

[6] B. K. H. Yen, A. Günther, M. A. Schmidt, K. F. Jensen, M. G. Bawendi, Angew. Chem. 2005, 117, 5583-5587;Angew. Chem. Int. Ed. 2005, 44, 5447-5451.

[7] H. Wang, X. Li, M. Uehara, Y. Yamaguchi, H. Nakamura, M. Miyazaki, H. Shimizu, H. Maeda, Chem. Commun. 2004, 48-49. 
[8] D. Shalom, R. C. R. Wootton, R. F. Winkle, B. F. Cottam, R. Vilar, A. J. deMello, C. P. Wilde, Mater. Lett. 2007, 61, 11461150 .

[9] A. Abou Hassan, O. Sandre, V. Cabuil, P. Tabeling, Chem. Commun. 2008, 1783-1785.

[10] L. Frenz, A. El Harrak, M. Pauly, S. Bégin-Colin, Andrew D. Griffiths, J.-C. Baret, Angew. Chem. 2008, 120, 6923-6926; Angew. Chem. Int. Ed. 2008, 47, 6817-6820.

[11] S. A. Khan, A. Gunther, M. A. Schmidt, K. F. Jensen, Langmuir 2004, 20, 8604-8611.

[12] B. F. Cottam, S. Krishnadasan, A. J. DeMello, J. C. DeMello, M. S. P. Shaffer, Lab Chip 2007, 7, 167-169.

[13] S. A. Khan, K. F. Jensen, Adv. Mater. 2007, 19, 2556-2560.

[14] J. F. Banfield, S. A. Welch, H. Zhang, T. T. Ebert, R. L. Penn, Science 2000, 289, 751-754.

[15] N. O. Nunez, M. P. Morales, P. Tartaj, C. J. Serna, J. Mater. Chem. 2000, 10, 2561-2565.

[16] G. J. Vroege, D. M. E. Thies-Weesie, A. V. Petukhov, B. J. Lemaire, P. Davidson, Adv. Mater. 2006, 18, 2565-2568.

[17] U. Schwertmann, E. Murad, Clays. Clay. Miner 1983, 31, $277-$ 292.

[18] M. P. Sharrock, R. E. Bodnar, J. Appl. Phys. 1985, 57, 3919-3924.

[19] B. J. Lemaire, P. Davidson, J. Ferré, J. P. Jamet, D. Petermann, P. Panine, I. Dozov, J. P. Jolivet, Europ. phys. J. E. 2004, 13, 291 308.

[20] A. Jahn, J. Reiner, W. Vreeland, D. DeVoe, L. Locascio, M. Gaitan, J. Nanopart. Res 2008, 10, 925-934.

[21] C.-H. Chang, B. Paul, V. Remcho, S. Atre, J. Hutchison, J. Nanopart. Res. 2008, 10, 965-980.
[22] Y. Song, J. Hormes, C. S. S. R. Kumar, Small 2008, 4, 698-711.

[23] J. B. Knight, A. Vishwanath, J. P. Brody, R. H. Austin, Phys. Rev. Lett. 1998, 80, 3863-3866.

[24] a) O. Levenspiel, in Chemical Reaction Engineering, $3^{\text {rd }}$ ed., Wiley, New York, 1999. b) C. A. Silebi, J.G. Dosramos, AIChE. j. 1989, 35, 1351-1364

[25] L. D. Scampavia, G. Blankenstein, J. Ruzicka, G. D. Christian, Analytical Chemistry 1995, 67, 2743-2749.

[26] D. Margulies, G. Melman, A. Shanzer, Nat. Mater. 2005, 4, 768771 .

[27] S. Krehula, S. Popovic, S. Music, Mater. Lett. 2002, 54, 108-113

[28] D.M.E. Thies-Weesie, JP. de Hoog, M.H. Hernandez Mendiola, A.V. Petukhov, G. J. Vroege, Chem. Mater. 2007, 19, 5538-5546 
Layout 1:

\section{Microfluidic}

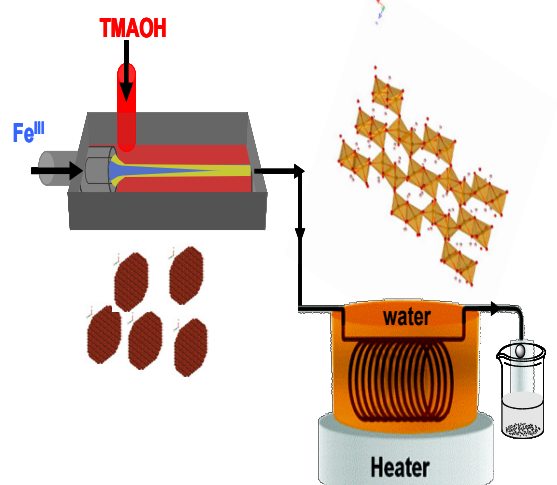

